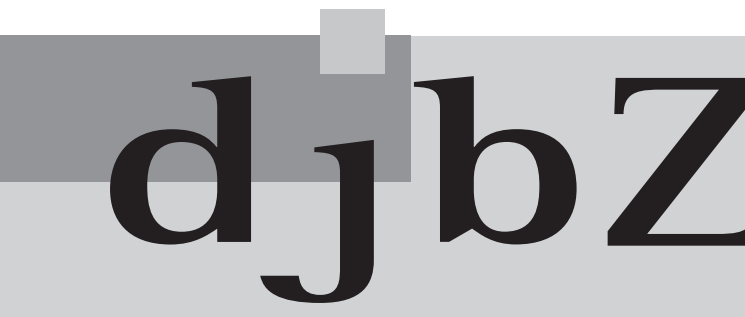

Zeitschrift des Deutschen

Juristinnenbundes

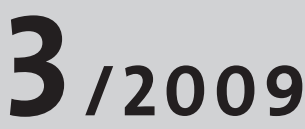

12. Jahrgang, Seite 101-166

Herausgeber: Deutscher Juristinnenbund e. V.

Präsidium: Jutta Wagner, Rechtsanwältin und Notarin, Fachanwältin für Familienrecht, Berlin (Präsidentin); Renate Maltry, Rechtsanwältin, Fachanwältin für Familienrecht und Erbrecht, München; Ramona Pisal, Vorsitzende Richterin am Oberlandesgericht, Brandenburg a.d.H. (Vizepräsidentinnen); Dagmar Brinkmann, Regierungsdirektorin, Frankfurt a.M. (Schatzmeisterin); Schriftleitung: Anke Gimbal, Rechtsassessorin, Geschäftsführerin Deutscher Juristinnenbund, Berlin.

\title{
Prozess der Individualisierung
}

In diesem Jahr ist der 60. Geburtstag unseres Grundgesetzes gefeiert worden. 60 Jahre Gleichberechtigung von Frauen und Männern in der Verfassung mit der Verpflichtung des Staates, auf den Abbau bestehender Nachteile zu Lasten der Frauen hinzuwirken. Mit 60 Jahren konnten berufstätige Frauen lange Zeit in Rente gehen. Zeit für den Ruhestand - auch für das Gleichberechtigungsgebot? Aufgabe erledigt, historischer Rückblick auf ein beeindruckendes „Lebenswerk“ von Art. 3 Abs. 2 Grundgesetz?

Lange Zeit war das für den Bereich soziale Sicherungssysteme und das Steuerrecht zu hoffen. Die unmittelbaren Diskriminierungen nach dem Geschlecht wurden abgeschafft. Als eine der letzten Bastionen fielen die Benachteiligungen nach dem Geschlecht (Kosten der Schwangerschaft und Geburt) bei den Beiträgen zur privaten Krankenkasse und zur inzwischen staatlich geförderten privaten Altersvorsorge. Das Verbot der mittelbaren Diskriminierung wegen des Geschlechts wurde europarechtlich erfunden und schließlich auch in der Verfassung entdeckt. Untersagt war damit die Verwendung von dem Anschein nach neutralen Vorschriften, Kriterien oder Verfahren, die bestimmte Personen aufgrund ihres Geschlechts ohne besondere sachliche Rechtfertigung benachteiligen. Die Umsetzung dieser Verpflichtung dauerte ebenfalls lange. Erst Mitte 2008 entschied das Bundesverfassungsgericht, dass die Benachteiligung von teilzeitbeschäftigten Beamtinnen beim Ruhegehalt mittelbar eine geschlechterdiskriminierende Wirkung im Sinne von Art. 3 GG hat und sie daher nichtig ist. Wichtig war auch das Fördergebot zur Durchsetzung der Gleichberechtigung und zum Abbau be- stehender Nachteile. Die eigenständige soziale Sicherung von Frauen und die Anerkennung weiblicher Erwerbsbiografien war in den 80er und auch noch in den 90er Jahren ein sozialpolitisches Trendthema. Die Kindererziehungszeiten in der gesetzlichen Rentenversicherung wurden eingeführt und ausgeweitet. Berufsrückkehrerinnen erhielten eigene Leistungsansprüche im Arbeitsförderungsrecht. Durch die Pflegeversicherung wurde die bisher unentgeltliche Arbeit von Töchtern und Schwiegertöchtern aufgewertet und sozial abgesichert. Tagesmütter wurden in die Unfallversicherung einbezogen. Der Bereich Rehabilitation und berufliche Eingliederung mit staatlicher Hilfe wurde massiv ausgeweitet. Im Steuerrecht diskutierte man über die Abschaffung des als traditionell überkommen angesehenen Ehegattensplittings. Institutionalisierte Frauen- und Gleichstellungsbeauftragte sollten auf den geschlechtergerechten Mitteleinsatz (neudeutsch Gender Budgeting) achten.

In den letzten Jahren hat sich dieser positive Trend zur Durchsetzung der tatsächlichen Gleichberechtigung in den sozialen Sicherungssystemen nicht nur deutlich verlangsamt, sondern teilweise umgekehrt. Unter dem Motto „Umbau der sozialen Sicherungssysteme wegen der demografischen Herausforderungen“ und "Stärkung des Versicherungsprinzips“ wurden solidarisch umverteilende Elemente gestrichen und bisher sozial abgesicherte Risiken privatisiert. Wie in den folgenden Beiträgen im Einzelnen dargestellt ist, betrifft dieser Trend nahezu alle Sicherungssysteme. Zuzahlungen und Leistungsausschlüsse im Krankenversicherungsrecht sind nichts anderes als Teilprivatisierungen, die Menschen mit geringerem Einkommen und

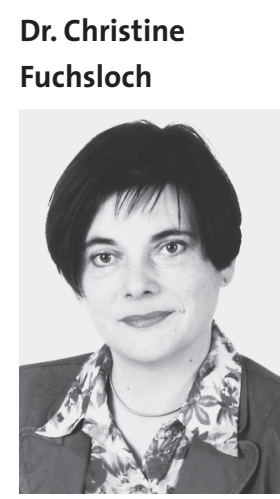

Mitglied der Kommission Recht der sozialen Sicherung, Familienlastenausgleich des djb; Richterin am LSG, Richterin des VerfG Brandenburg, Potsdam 
damit oft Frauen besonders betreffen. Von den bis 1992 geltenden Mindestentgelten bei der Rentenberechnung profitierten vor allem Frauen mit typischerweise niedrigerem Einkommen. Wenn die gesetzliche Altersrente im Umlagesystem auf der Grundlage des Dreigenerationenvertrages durch eine private kapitalgedeckte Altersrente ergänzt werden muss, so können sich das beispielsweise Alleinerziehende trotz staatlichem Zuschuss oft nicht oder nur in bescheidenem Umfang leisten, wodurch deren Altersarmut vorprogrammiert ist. Im Rentenversicherungsrecht sind jedoch bei der gesetzlichen Rente Strukturveränderungen nötig und möglich, die zu einer eigenständigen Alterssicherung von Frauen führen. Nirgends ist die Privatisierung und Individualisierung von sozialen Risiken so deutlich wie beim Risiko der Arbeitslosigkeit. Durch die drastische Verkürzung des Anspruchs auf die Versicherungsleis- tung Arbeitslosengeld greift bereits in der Regel nach zwölf Monaten Arbeitslosigkeit nur noch das Leistungssystem Hartz IV. Im Verhältnis zur „alten“ Arbeitslosenhilfe zeichnet sich dieses Leistungssystem durch eine verschärfte Anrechnung des Partnereinkommens aus, wodurch die Frauen ohne eigenständigen Leistungsanspruch auf die „Versorgerehe“ bzw. „Versorgerlebensgemeinschaft" verwiesen werden. Auch innerhalb des Leistungssystems Hartz IV gibt es diverse geschlechtsspezifische Verfestigungen (z. B. durch die Ausweitung von frauentypischen Minijobs als zumutbarer Beschäftigungsalternative) und durch die Art der Einkommensberechnung.

Die nachfolgenden Beiträge zu den einzelnen Leistungssystemen und zum Steuerrecht zeigen Handlungsbedarf und zwar auch nach 60 Jahren Grundgesetz und damit 60 Jahren verfassungsrechtlichem Gleichberechtigungsgebot.

\section{Der Grundsatz der Gleichberechtigung von Frauen und Männern im Einkommensteuerrecht}

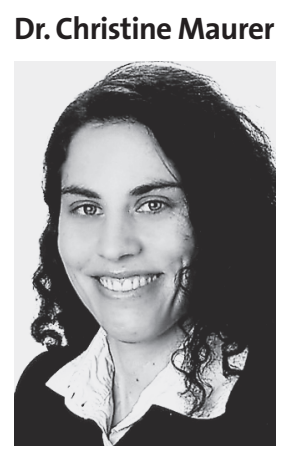

Mitglied der Kommission Recht der sozialen Sicherung, Familienlastenausgleich des djb; Rechtsanwältin, München

\section{Einleitung}

Auch im Einkommensteuerrecht gilt der Grundsatz der Gleichberechtigung von Frauen und Männern. Die besonderen Gleichheitssätze in Art. 3 Abs. 2 und 3 GG konkretisieren und verstärken den allgemeinen Gleichheitssatz in Art. 3 Abs. 1 GG. Sie konkretisieren daher auch die Steuergerechtigkeit im Hinblick auf geschlechtsbezogene Benachteiligungen und ziehen der Gestaltungsfreiheit des Gesetzgebers engere Grenzen. Dabei kommt dem Verbot der mittelbaren Diskriminierung besondere Bedeutung zu, weil das Steuerrecht unter anderem an geschlechtsbezogene Erwerbsmuster und Arbeitsmarktstrukturen sowie familiäre Rollenverteilungen anknüpft. Dadurch können Benachteiligungen verfestigt oder sogar verstärkt werden. Der Gesetzgeber ist daher gefordert, auf eine konsequente Umsetzung des Verbots der Benachteiligung im Einkommensteuerrecht hinzuwirken. Zentrale Kritikpunkte des djb sind nach wie vor das Ehegattensplitting, das Lohnsteuerverfahren für verheiratete Eheleute und die Absetzbarkeit von Kinderbetreuungskosten. Zudem zeigt sich, dass für eine Bewertung der realen Wirkungen der Besteuerung geschlechterdifferenzierte Statistiken auch im Steuerrecht notwendig sind.

\section{Reform der Ehebesteuerung}

Der djb fordert seit jeher die Abschaffung des Ehegattensplittings im Einkommensteuerrecht $(\mathbb{S} \mathbb{S}$ 26b, 32a Abs. 5 EStG). Die Kritik am Ehegatten- splitting betrifft vor allem die negativen Erwerbsanreize für Frauen ${ }^{1}$. Mit einer „zusätzlichen“ Berufstätigkeit der Ehefrau geht der Splittingvorteil verloren, so dass sich eine nicht nur geringfügige Berufstätigkeit erst lohnt, wenn der Splittingvorteil zumindest zurückverdient wird. Das Splittingvolumen fließt zu 93 Prozent in die alten Bundesländer. ${ }^{2}$ Diese Verteilung zeigt die Abhängigkeit des Splittingvorteils von geschlechtsbezogenen Erwerbsmustern und Arbeitsmarktstrukturen. In den neuen Bundesländern sind Frauen dabei nicht nur häufiger vollzeiterwerbstätig. Auch die Lohndifferenzen zwischen Frauen und Männern (gender pay gap) sind geringer. Gerade diese geringere Differenz senkt aber auch den Splittingvorteil. Das Ehegattensplitting konterkariert damit das Ziel, Lohndifferenzen abzubauen. Bei Arbeitnehmenden verstärkt die Lohnsteuerklasse $\mathrm{V}$ die Benachteiligung verheirateter Frauen durch die ungleiche Verteilung der Lohnsteuer zwischen den Eheleuten. Letztlich ist damit der vom Bundesverfassungsgericht bereits 1957 verbotene Edukationseffekt erhalten geblieben. ${ }^{3}$ Das Ehegatten-

1 Vgl. Hintergrundpapier 06-15 zur aktuellen Diskussion über eine Reform der Besteuerung von Ehe und Familie.

2 Bach, Stefan et al. (2003): Untersuchung zu den Wirkungen der gegenwärtigen Ehegattenbesteuerung auf Grundlage der fortgeschriebenen Einzeldaten der Einkommensteuerstatistik, Materialen des DIW Berlin, Nr. 27, S. 45 f.

3 Vgl. BVerfGE 6, 55 . 